July 22,2016

\title{
HARMONIC MAPS AND THE SCHOEN CONJECTURE
}

\author{
VLADIMIR MARKOVIC
}

\begin{abstract}
We show that every quasisymmetric homeomorphism of the circle $\partial \mathbb{H}^{2}$ admits a harmonic quasiconformal extension to the hyperbolic plane $\mathbb{H}^{2}$. This proves the Schoen Conjecture.
\end{abstract}

\section{INTRODUCTION}

1.1. Statements of Results. A central question in the theory of harmonic maps is when the homotopy class (with prescribed boundary values) of a map $F: M \rightarrow N$ between two negatively curved Riemannian manifolds contains a harmonic map. When $F$ has finite total energy the existence and uniqueness of such harmonic maps is known (for example see [12]).

When $F$ does not have finite total energy the problem is harder and much less is known. The case that has been studied the most is when $M$ and $N$ are both equal to the hyperbolic space $\mathbb{H}^{n}$. In [11] Schoen made the following conjecture which has been driving this subject since.

Conjecture 1 (Schoen). Every quasisymmetric homeomorphism $u: \partial \mathbb{H}^{2} \rightarrow$ $\partial \mathbb{H}^{2}$ admits a unique harmonic quasiconformal extension $f: \mathbb{H}^{2} \rightarrow \mathbb{H}^{2}$.

The uniqueness part of this conjecture was established in [7] by $\mathrm{Li}$ and Tam. In [7] and [8] they proved that every diffeomorphism $u: \partial \mathbb{H}^{2} \rightarrow$ $\partial \mathbb{H}^{2}$ admits a harmonic quasiconformal extension to $\mathbb{H}^{2}$. There exists an extensive literature about this topic (for example, see [15], [14], [6], [1], [9], for further results related to the existence part of the conjecture).

Remark. The 3-dimensional version of this conjecture has been settled in [10]. But the methods used in that argument rely on the quasiconformal rigidity in higher dimensions and are not applicable to the 2 dimensional situation.

A harmonic quasiconformal map $f: \mathbb{H}^{2} \rightarrow \mathbb{H}^{2}$ is determined by its boundary values which are given by a quasisymmetric homeomorphism $u: \partial \mathbb{H}^{2} \rightarrow$ $\partial \mathbb{H}^{2}$. However, quantifying how $f$ depends on $u$ is difficult. To illustrate the subtlety of this issue in [10] we constructed a sequence of diffeomorhisms $u_{n}: \partial \mathbb{H}^{2} \rightarrow \partial \mathbb{H}^{2}$, that converge to the identity in the $C^{0}$ sense but the corresponding harmonic quasiconformal extensions degenerate on compact sets in $\mathbb{H}^{2}$ and we can not extract any sort of limit (this behavior is very

2000 Mathematics Subject Classification. Primary 53C43.

This work was partially supported by the Simons Investigator Award 409745 from the Simons Foundation and by the NSF grant number DMS-1201463 
different than in the case of harmonic functions on $\mathbb{H}^{2}$, where the boundary map effectively controls the behavior of a harmonic function inside the disc).

Remark. When we write $C=C\left(K_{1}, K_{2}, \ldots\right)$, we mean that the constant $C$ depends only on $K_{1}, K_{2}, \ldots$. We use this policy throughout the paper.

In the remainder of this paper we prove the following theorem which takes care of the main difficulty we described above.

Theorem 1.1. For every $K>1$ there exists a constant $C=C(K)$ with the following properties. We let $g: \mathbb{H}^{2} \rightarrow \mathbb{H}^{2}$ be any $K$-quasiconformal homeomorphism and assume that there exists a harmonic quasiconformal homeomorphism $f: \mathbb{H}^{2} \rightarrow \mathbb{H}^{2}$ that agrees with $g$ on the boundary $\partial \mathbb{H}^{2}$. Then

$$
\sup _{z \in \mathbb{H}^{2}} d(g(z), f(z)) \leq C,
$$

where $d(\cdot, \cdot)$ denotes the hyperbolic distance.

We say that a homeomorphism $u: \partial \mathbb{H}^{2} \rightarrow \partial \mathbb{H}^{2}$ is $K$-quasisymmetric if it admits a $K$-quasiconformal extension to $\mathbb{H}^{2}$. Theorem 1.1 implies that the set of $K$-quasisymmetric homeomorphisms of $\partial \mathbb{H}^{2}$ that admit harmonic quasiconformal extensions is closed with respect to pointwise convergence. That is, if $u_{n}$ is a sequence of $K$-quasisymmetric maps that admit harmonic and quasiconformal extensions, and if $u_{n}$ pointwise converges to (a necessarily) $K$-quasisymmetric map $u$, then $u$ also admits a harmonic quasiconformal extension (this is a standard argument and it follows from Cheng's lemma [2] and the Azrela-Ascoli theorem).

The following theorem is an immediate corollary of Theorem 1.1 and the result of $\mathrm{Li}$ and Tam [7] that every diffeomorphism of $\partial \mathbb{H}^{2}$ admits a harmonic quasiconformal extension. It is a well known fact that every $K$-quasisymmetric homeomorphism of $\partial \mathbb{H}^{2}$ is a pointwise limit of $K$ quasisymmetric diffeomorphisms.

Theorem 1.2. Every quasisymmetric map of $\partial \mathbb{H}^{2}$ admits a harmonic quasiconformal extension to $\mathbb{H}^{2}$.

The theory of harmonic maps between hyperbolic surfaces is closely related to Teichmüller theory. A corollary of Theorem 1.2 is that the Teichmüller space of any Riemann surface $S$ (including the Universal Teichmüller space) can be parametrized by the space of bounded holomorphic quadratic differentials by taking the Hopf differential of the harmonic mapping representing a given homotopy class (see [14]). In particular, we have the following corollary of the uniqueness result of $\mathrm{Li}$ and Tam and Theorem 1.2 .

Theorem 1.3. A quasiconformal map between any two complete hyperbolic Riemann surfaces is isotopic to a unique harmonic quasiconformal map. 
1.2. The Unit Disc Model of the Hyperbolic Plane and Harmonic Maps. We identify the hyperbolic plane $\mathbb{H}^{2}$ with the unit disc $\mathbb{D}$ in the complex plane and the boundary $\partial \mathbb{H}^{2}$ with the unit circle $\mathbb{S}^{1}$. The density of the hyperbolic metric is

$$
\sigma(z)=\frac{2}{1-|z|^{2}}, \quad z \in \mathbb{D} .
$$

A $C^{2}$ map $f: \mathbb{D} \rightarrow \mathbb{D}$ is harmonic if it satisfies the equation

$$
f_{z \bar{z}}+2\left(\frac{\sigma_{w}}{\sigma} \circ f\right) f_{z} f_{\bar{z}}=0 .
$$

For a harmonic map $f$ by

$$
\operatorname{Hopf}[f]=\left(\sigma^{2} \circ f\right) f_{z} f_{\bar{z}},
$$

we denote the Hopf Differential. Then Hopf $[f]$ is a holomorphic function on D. We let

$$
\mathrm{BQD}(\mathbb{D})=\left\{\Phi \text { holomorphic on } \mathbb{D}: \sup _{z \in \mathbb{D}} \sigma^{-2}(z)|\Phi(z)|=\|\Phi\|_{\infty} \leq \infty\right\}
$$

denote the space of bounded holomorphic quadratic functions on $\mathbb{D}$. Wan showed in $[15]$ that a harmonic map $f: \mathbb{D} \rightarrow \mathbb{D}$ is quasiconformal if and only if $\operatorname{Hopf}[f] \in \mathrm{BQD}(\mathbb{D})$.

1.3. The Polar Coordinates and the Laplacian of the Distance Function. Each $z \in \mathbb{D}$ is written in Euclidean polar coordinates as $z=r e^{i \theta}$ for some $(r, \theta) \in[0,1) \times(-\pi, \pi]$. We also introduce the geodesic polar coordinates $(\rho, \theta) \in[0, \infty) \times(-\pi, \pi]$. If $z \in \mathbb{D}$ has Euclidean polar coordinates $(r, \theta)$ then its geodesic polar coordinates are $(\rho, \theta)$, where $\rho(z)=\rho(r)=d(z, 0)$ denotes the distance with respect to the hyperbolic metric.

The harmonic maps equation can be expressed with respect to the geodesic polar coordinates. To simplify notation in the rest of the text we let

$$
\boldsymbol{\rho}=\rho \circ f, \text { and } \boldsymbol{\theta}=\theta \circ f
$$

for a harmonic map $f$. Assuming this notation, we state the following well known equation (for example see formula (6.1) in [5])

$$
\Delta \boldsymbol{\rho}(z)=\frac{1}{2} \sinh (2 \boldsymbol{\rho}(z))|\nabla \boldsymbol{\theta}(z)|^{2}, \text { when } \boldsymbol{\rho}(z) \neq 0,
$$

where $\Delta$ and $\nabla$ denote respectively the Laplacian and the gradient computed with respect the hyperbolic metric.

Remark. In [5] Han computed the above formula for $\Delta \rho(z)$ with respect to the Euclidean metric on the z-plane. Our formula follows from Han's by dividing it by $\sigma^{2}(z)$. 
Although the function $\boldsymbol{\rho}$ is not $C^{2}$ at the point where $\boldsymbol{\rho}(z)=0$, the function $\rho^{2}$ is $C^{2}$ everywhere on $\mathbb{D}$. We compute

$$
\Delta \boldsymbol{\rho}^{2}=\frac{1}{4 \sigma^{2}}\left(\boldsymbol{\rho}^{2}\right)_{z \bar{z}}=\frac{\boldsymbol{\rho}_{z} \boldsymbol{\rho}_{\bar{z}}}{2 \sigma^{2}}+2 \boldsymbol{\rho} \Delta \boldsymbol{\rho}=\frac{\left|\boldsymbol{\rho}_{z}\right|^{2}}{2 \sigma^{2}}+2 \boldsymbol{\rho} \Delta \boldsymbol{\rho},
$$

since $\boldsymbol{\rho}$ is real valued and $\overline{\left(\overline{\boldsymbol{\rho}}_{z}\right)}=\boldsymbol{\rho}_{\bar{z}}$. The inequality

$$
\Delta \boldsymbol{\rho}^{2}(z) \geq \boldsymbol{\rho}(z) \sinh (2 \boldsymbol{\rho}(z))|\nabla \boldsymbol{\theta}(z)|^{2}, \text { for every } z \in \mathbb{D},
$$

follows.

The first observation that follows from (1) is that $\rho^{2}$ is subharmonic. In fact, the main idea behind the argument (which is explained below) comes from comparing this inequality and the Green's formula we state next.

1.4. The Green's Formula. We state the following corollary of the second Green's Identity. This has been proved as Theorem 4.2 in [13] (see also [10] and references therein). We sketch the proof for the sake of completeness.

Proposition 1.1. For a $C^{2}$ function $F$ on $\mathbb{D}$, the following holds true

$$
F(0)+\frac{2}{\pi} \int_{r \mathbb{D}} \Delta F(z) \log \frac{r}{|z|} \frac{d x d y}{\left(1-|z|^{2}\right)^{2}}=\frac{1}{2 \pi} \int_{\mathbb{S}^{1}} F(z)|d z|,
$$

where $z=x+i y$, and $\Delta F$ is the Laplacian of $F$ (computed with respect to the hyperbolic metric).

Proof. Recall that $\frac{1}{2} \log (r /|z|)=\mathbf{g}_{r}(z, 0)$, where $\mathbf{g}_{r}(\cdot, \cdot)$ is the Green's function on the product $r \mathbb{D} \times r \mathbb{D}$. Let $\epsilon>0$. By the second Green's Identity applied to the functions $F$ and $\mathbf{g}_{r}$ on the annulus $0<\epsilon \leq|z| \leq r$, we find

$$
\begin{gathered}
\int_{r \mathbb{D} \backslash \in \mathbb{D}} \Delta F(z) \mathbf{g}_{r}(z, 0) \frac{4 d x d y}{\left(1-|z|^{2}\right)^{2}}=\int_{|z|=\epsilon} \mathbf{g}_{r}(z, 0) \frac{\partial F}{\partial \vec{n}}(z) \frac{2|d z|}{\left(1-\epsilon^{2}\right)} \\
-\int_{|z|=r} F(z) \frac{\partial \mathbf{g}_{r}}{\partial \vec{n}}(z, 0) \frac{2|d z|}{\left(1-r^{2}\right)}-\int_{|z|=\epsilon} F(z) \frac{\partial \mathbf{g}_{r}}{\partial \vec{n}}(z, 0) \frac{2|d z|}{\left(1-\epsilon^{2}\right)},
\end{gathered}
$$

where $\frac{\partial}{\partial \vec{n}}$ is the derivative along the unit (with respect to the hyperbolic metric) outward pointing vector that is normal to the corresponding oriented boundary component. We replace $\frac{1}{2} \log (r /|z|)=\mathbf{g}_{r}(z, 0)$, and explicitly compute its partial derivative along $\vec{n}$. The lemma follows by letting $\epsilon \rightarrow$ 0 .

Next, we express this formula in the Euclidean polar coordinates. This is the version we will use. Set

$$
\mathcal{R}(r)=[0, r] \times(-\pi, \pi] .
$$


Then

$$
F(0)+\frac{2}{\pi} \int_{\mathcal{R}(r)} t \Delta F\left(t e^{i \theta}\right) \log \frac{r}{t} \frac{d t d \theta}{\left(1-t^{2}\right)^{2}}=\frac{1}{2 \pi} \int_{-\pi}^{\pi} F\left(r e^{i \theta}\right) d \theta .
$$

1.5. The Normalizations. Once and for all fix $K>1$. In the remainder of this paper $g: \mathbb{D} \rightarrow \mathbb{D}$ denotes an arbitrary $K$-quasiconformal homeomorphism that happens to share the same boundary values as a harmonic quasiconformal homeomorphisms of $\mathbb{D}$. We will denote this harmonic map by $f: \mathbb{D} \rightarrow \mathbb{D}$. In addition (after simultaneously precomposing and postcompising $f$ and $g$ by suitable Möbis transformations of $\mathbb{D}$ ) we may suppose the following.

- The distance between $f$ and $g$ is nearly achieved at the origin, that is

$$
\sup _{z \in \mathbb{D}} d(f(z), g(z)) \leq d(f(0), g(0))+1 .
$$

- The map $g$ fixes the origin, that is $g(0)=0$, and also $f$ maps the origin to the segment $[0,1)$, that is $0 \leq f(0)<1$.

- The distance between $f(0)$ and $g(0)=0$ is at least 1 , that is $\boldsymbol{\rho}(0) \geq 1$ (if $\boldsymbol{\rho}(0)<1$ then Theorem 1.1 holds for such $g$ and $f$ ).

These assumptions are standing in the remainder of this article.

1.6. The Main Idea. We are left with the task of finding an upper bound of $\boldsymbol{\rho}(0)$ (that depends only on $K$ ). We choose to analyze the distance function $\boldsymbol{\rho}(z), z \in \mathbb{D}$, with the assumption that $\boldsymbol{\rho}(0)$ is close to being the supremum of the distance between $f$ and a $K$-quasiconformal map $g$. The benefit of making this choice is that we can a-priori bound from above the growth of $\boldsymbol{\rho}(z)$.

Lemma 1.1. For any $z \in \mathbb{D}$ the inequality

$$
\boldsymbol{\rho}^{2}(z) \leq \boldsymbol{\rho}^{2}(0)+3 K^{2} \boldsymbol{\rho}(0)(\rho(z)+4)^{2}
$$

holds.

Proof. It was proved (see Theorem 5.1 in [3]) that every $K$-quasiconformal homeomorphism is a $(K, 3 K)$ quasi-isometry in terms of the hyperbolic metric. Applying this to the map $g$ we derive the inequality

$$
d(g(z), 0)=d(g(z), g(0)) \leq K \rho(z)+3 K .
$$

By the triangle inequality and using the previous estimate we get

$\boldsymbol{\rho}(z)=d(0, f(z)) \leq d(0, g(z))+d(f(z), g(z)) \leq(K \rho(z)+3 K)+(\boldsymbol{\rho}(0)+1)$.

Squaring the above inequality (and since $K \geq 1$ ) we obtain

$\boldsymbol{\rho}^{2}(z) \leq(\boldsymbol{\rho}(0)+(K \rho(z)+4 K))^{2}=\boldsymbol{\rho}^{2}(0)+2 K \boldsymbol{\rho}(0)(\rho(z)+4)+K^{2}(\rho(z)+4)^{2}$.

Replacing the inequalities $(\rho(z)+4)<(\rho(z)+4)^{2}, \boldsymbol{\rho}(0) \geq 1$ and $K \leq K^{2}$, in the previous inequality yields the lemma. 
Remark. The conclusion of the lemma is that $\rho^{2}(z)$ grows at a bounded (in terms of $K$ ) rate. In fact, the growth of the quotient $\boldsymbol{\rho}^{2}(z) / \boldsymbol{\rho}^{2}(0)$ is negligible when $\rho^{2}(z)$ is small comparing to $\boldsymbol{\rho}(0)$.

Combining this with the Green's identity (3) we can bound the integral of $\Delta \boldsymbol{\rho}^{2}$ as follows

$$
\frac{2}{\pi} \int_{\mathcal{R}(r)} t \Delta \boldsymbol{\rho}^{2}\left(t e^{i \theta}\right) \log \frac{r}{t} \frac{d t d \theta}{\left(1-t^{2}\right)^{2}} \leq 3 K^{2} \boldsymbol{\rho}(0)(\rho(r)+4)^{2} .
$$

On the other hand, the inequality (1) suggests the estimate

$$
\int_{\mathcal{R}(r)} t \Delta \boldsymbol{\rho}^{2}\left(t e^{i \theta}\right) \log \frac{r}{t} \frac{d t d \theta}{\left(1-t^{2}\right)^{2}} \geq O(\boldsymbol{\rho}(0) \sinh (2 \boldsymbol{\rho}(0))),
$$

when $r$ stays bounded (in terms of $K$ ). Combining the previous two inequalities suggests the estimate

$$
\sinh (2 \boldsymbol{\rho}(0)) \leq O(\rho(r))^{2} .
$$

Thus, in order to prove Theorem 1.1 it would suffice to show that such an estimate actually holds for some $r=r(K)$. We do just that in the rest of the paper.

1.7. The Outline and Organization. Now we briefly mention the main steps in the proof and the motivation behind them. The main result of Section 2 is the estimate

$$
\int_{E_{r^{2}}}\left(\left|\boldsymbol{\theta}\left(r^{2} e^{i \theta}\right)\right|-\left|\boldsymbol{\theta}\left(e^{i \theta} / 2\right)\right|\right)^{2} d \theta \leq \frac{\Psi(r, K)}{\sinh (\boldsymbol{\rho}(0))},
$$

which holds for every $r \in(1 / \sqrt{2}, 1)$ and where $\Psi(r, K)$ is a function which we will compute explicitly. Here $E_{r}$ is the set of $\theta$ 's for which $\rho^{2}\left(t e^{i \theta}\right)$ does not dip below $\rho^{2}(0) / 4$ for any $0 \leq t \leq r$. The only ingredients that are used to prove (4) are the Green's formula and the lower bound (1) on $\Delta \boldsymbol{\rho}^{2}$ that we stated above. The inequality (4) can be rewritten in the form

$$
\sinh (\boldsymbol{\rho}(0)) \leq \frac{\Psi(r, K)}{\int_{E_{r^{2}}}\left(\left|\boldsymbol{\theta}\left(r^{2} e^{i \theta}\right)\right|-\left|\boldsymbol{\theta}\left(e^{i \theta} / 2\right)\right|\right)^{2} d \theta},
$$

which explains the motivation behind proving it. Namely, this inequality gives us a way of estimating $\boldsymbol{\rho}(0)$ from above (only in terms of $K$ ) which is the content of the remainder of this paper.

In order to complete the proof of the main theorem, it is clear that the remaining step is to bound from below the integral on the left hand side of (4) for some $r=r(K)$. To do this we first need to find a lower bound for 
$\left|\boldsymbol{\theta}\left(r e^{i \theta}\right)\right|$. In Section 3 we find such a bound for $\theta \in U_{r}$, where $U_{r}$ is the set of $\theta$ 's for which $\boldsymbol{\rho}^{2}\left(r e^{i \theta}\right)$ is relatively close to $\boldsymbol{\rho}^{2}(0)$, that is when

$$
\boldsymbol{\rho}^{2}\left(r e^{i \theta}\right) \geq \boldsymbol{\rho}^{2}(0)-K^{2} \boldsymbol{\rho}(0)(\rho(r)+4)^{2} .
$$

Remark. Note that for $\theta$ to be in $U_{r}$ we do not require that the defining inequality holds on the entire segment $\left[0, r e^{i \theta}\right)$, which we do for $\theta \in E_{r}$. This is why it will be more demanding to estimate below the measure of $E_{r}$ than the measure of $U_{r}$.

When $\theta \in U_{r}$, then for $\rho(r)$ large we will show that $\boldsymbol{\theta}\left(r e^{i \theta}\right)$ is very close to $(\theta \circ g)\left(r e^{i \theta}\right)$ (this will follow from the assumption that the distance between $f$ and $g$ is nearly maximized at 0$)$. This will yield the estimate

$$
\left|\boldsymbol{\theta}\left(r e^{i \theta}\right)\right| \geq \frac{\left|e^{i \theta}-e^{i \theta_{0}}\right|^{K}}{16^{K}}-o(1-r), \quad \theta \in U_{r},
$$

where the constants standing behind the notation $o(1-r)$ depend only on $K$. This estimate, together with the upper bound on $\boldsymbol{\theta}\left(e^{i \theta} / 2\right)$ (which we discuss below), then yields the integral estimate

$$
\int_{T}\left(\left|\boldsymbol{\theta}\left(r e^{i \theta}\right)\right|-\left|\boldsymbol{\theta}\left(e^{i \theta} / 2\right)\right|\right)^{2} d \theta \geq q=q(K)>0,
$$

for every measurable set $T \subset U_{r}$, of measure at least $\frac{\pi}{4}$ and for every $r \geq$ $r_{0}(K)$. This gets us closer to the desired lower bound on the integral on the left hand side in (4).

In order to apply (5) to estimate from below the integral in (4) it remains to prove that the set $U_{r} \cap E_{r}$ has measure at least $\pi / 4$. We show this is true when $\boldsymbol{\rho}(0)$ is large enough. In Section 4 we prove the following inequality

$$
\sup _{z \in \mathbb{D}}|\partial f|(z) \leq C \sup _{z \in \mathbb{D}} d(f(z), g(z))+B(K),
$$

where $C$ is a universal constant and $B(K)$ depends only on $K$. This inequality follows readily from the following two cornerstones in this subject. The first is Wolf's work [16] in which he accurately describes the image $f(\gamma)$, where $\gamma$ is a segment of a horizontal trajectory of $\operatorname{Hopf}[f]$ that is away from a zero of Hopf $[f]$. The second is the Wan's result [15] that provides an upper bound of $|\partial f|$ in terms of $\|\operatorname{Hopf}[f]\|_{\infty}$.

We then use inequality (6) in Section 5 to finish the proof of Theorem 1.1. First we use (6) to find an upper bound for $\boldsymbol{\theta}\left(e^{i \theta} / 2\right)$, when $\theta \in E_{1 / 2}$. We prove that $\boldsymbol{\theta}\left(e^{i \theta} / 2\right)$ is smaller than any $\epsilon>0$ providing that $\boldsymbol{\rho}(0)$ is large enough. Also, using (6) we show that the set $E_{r}$ has almost the full measure in $(-\pi, \pi]$ when $\boldsymbol{\rho}(0)$ is large enough. In particular we show that the set $U_{r} \cap E_{r}$ has measure at least $\pi / 4$ when $\boldsymbol{\rho}(0)$ is large. This is enough to show from (4) and (5) that

$$
\sinh (\boldsymbol{\rho}(0)) \leq \frac{\Psi\left(r_{0}(K), K\right)}{q(K)}
$$


holds.

1.8. Acknowledgment. I thank the referee and Yunhui $\mathrm{Wu}$ for comments and corrections.

\section{Estimating $\int \Delta \boldsymbol{\rho}^{2}$}

The purpose of this subsection is to derive a lower bound for the integral of $\Delta \boldsymbol{\rho}^{2}$ on the disc $r \mathbb{D}$ for certain $r \in(0,1)$ and then use it to establish the estimate (4) from the outline above . Our result is summarized in Lemma 2.1 below.

For every $\theta$ we derive the inequality

$$
\left|\boldsymbol{\theta}\left(r e^{i \theta}\right)\right| \leq\left|\boldsymbol{\theta}\left(e^{i \theta} / 2\right)\right|+\int_{\frac{1}{2}}^{r}\left|\nabla \boldsymbol{\theta}\left(t e^{i \theta}\right)\right| \frac{2 d t}{1-t^{2}} .
$$

Set

$$
\Theta\left(r e^{i \theta}\right)=|| \boldsymbol{\theta}\left(r e^{i \theta}\right)|-| \boldsymbol{\theta}\left(e^{i \theta} / 2\right)|| .
$$

Then (7) becomes

$$
\Theta\left(r e^{i \theta}\right) \leq \int_{\frac{1}{2}}^{r}\left|\nabla \boldsymbol{\theta}\left(t e^{i \theta}\right)\right| \frac{2 d t}{1-t^{2}}
$$

The Cauchy-Schwarz inequality yields

$$
\Theta^{2}\left(r e^{i \theta}\right) \leq 4 \int_{\frac{1}{2}}^{r}\left|\nabla \boldsymbol{\theta}\left(t e^{i \theta}\right)\right|^{2} \frac{d t}{\left(1-t^{2}\right)^{2}},
$$

and furthermore we obtain

$$
\Theta^{2}\left(r e^{i \theta}\right) \leq 8 \int_{\frac{1}{2}}^{r} t\left|\nabla \boldsymbol{\theta}\left(t e^{i \theta}\right)\right|^{2} \frac{d t}{\left(1-t^{2}\right)^{2}}, \quad \text { for every } \quad \frac{1}{2} \leq r<1 .
$$

Remark. We want to relate the integral on the right hand side of (9) to the surface integral in the Green's formula (3). However, this integral in (3) is of the form $\int t \log (r / t) \Delta \boldsymbol{\rho}^{2}\left(t e^{i \theta}\right)$. This explains why we multiplied the term $\left|\nabla \boldsymbol{\theta}\left(t e^{i \theta}\right)\right|^{2}$ by $t$ and why we had to integrate from $1 / 2$ to $r$ in (7) (and not from 0 to $r$ ).

To further estimate the integral on the right hand side we restrict to a certain set of $\theta$ 's.

Definition 2.1. Fix $0<r<1$. We let

$$
E_{r}=\left\{\theta \in(-\pi, \pi]: \boldsymbol{\rho}^{2}\left(t e^{i \theta}\right) \geq \frac{\boldsymbol{\rho}^{2}(0)}{4} \text { for every } 0 \leq t \leq r\right\} .
$$


Assuming $\theta \in E_{r}$ we get

$$
\int_{\frac{1}{2}}^{r} \frac{t\left|\nabla \boldsymbol{\theta}\left(t e^{i \theta}\right)\right|^{2} d t}{\left(1-t^{2}\right)^{2}} \leq \frac{2}{\boldsymbol{\rho}(0) \sinh (\boldsymbol{\rho}(0))} \int_{\frac{1}{2}}^{r} \boldsymbol{\rho}\left(t e^{i \theta}\right) \sinh \left(2 \boldsymbol{\rho}\left(t e^{i \theta}\right)\right) \frac{t\left|\nabla \boldsymbol{\theta}\left(t e^{i \theta}\right)\right|^{2} d t}{\left(1-t^{2}\right)^{2}} .
$$

Recall the inequality (1)

$$
\Delta \boldsymbol{\rho}^{2}(z) \geq \boldsymbol{\rho}(z) \sinh (2 \boldsymbol{\rho}(z))|\nabla \boldsymbol{\theta}(z)|^{2}, \text { for every } z \in \mathbb{D},
$$

which provides the lower bound for the Laplacian $\Delta \boldsymbol{\rho}^{2}$. Replacing (1) in the previous inequality we obtain

$$
\int_{\frac{1}{2}}^{r} \frac{t\left|\nabla \boldsymbol{\theta}\left(t e^{i \theta}\right)\right|^{2} d t}{\left(1-t^{2}\right)^{2}} \leq \frac{2}{\boldsymbol{\rho}(0) \sinh (\boldsymbol{\rho}(0))} \int_{\frac{1}{2}}^{r} \frac{t \Delta \boldsymbol{\rho}^{2}\left(t e^{i \theta}\right) d t}{\left(1-t^{2}\right)^{2}}, \quad \theta \in E_{r} .
$$

Putting (9) and (10) together we get for every $1 / 2 \leq r<1$ the following inequality

$$
\Theta^{2}\left(r e^{i \theta}\right) \leq \frac{16}{\boldsymbol{\rho}(0) \sinh (\boldsymbol{\rho}(0))} \int_{\frac{1}{2}}^{r} t \Delta \boldsymbol{\rho}^{2}\left(t e^{i \theta}\right) \frac{d t}{\left(1-t^{2}\right)^{2}}, \quad \theta \in E_{r} .
$$

Integrating both sides of the inequality (11) over the set $E_{r}$ we find that

$$
\int_{E_{r}} \Theta^{2}\left(r e^{i \theta}\right) d \theta \leq \frac{16}{\boldsymbol{\rho}(0) \sinh (\boldsymbol{\rho}(0))} \int_{\left[\frac{1}{2}, r\right] \times E_{r}} t \Delta \boldsymbol{\rho}^{2}\left(t e^{i \theta}\right) \frac{d t d \theta}{\left(1-t^{2}\right)^{2}},
$$

and since $t \Delta \boldsymbol{\rho}^{2}\left(t e^{i \theta}\right) \geq 0$, for $1 / 2 \leq r<1$ we derive

$$
\int_{E_{r}} \Theta^{2}\left(r e^{i \theta}\right) d \theta \leq \frac{16}{\boldsymbol{\rho}(0) \sinh (\boldsymbol{\rho}(0))} \int_{\mathcal{R}(r)} t \Delta \boldsymbol{\rho}^{2}\left(t e^{i \theta}\right) \frac{d t d \theta}{\left(1-t^{2}\right)^{2}},
$$

where $\mathcal{R}(r)=[0, r] \times(-\pi, \pi]$.

We still need to insert the $\log (r / t)$ term in the integral on the right hand side of (12). Replacing $r$ by $r^{2}$ in (12) we get the estimate

$$
\int_{E_{r^{2}}} \Theta^{2}\left(r^{2} e^{i \theta}\right) d \theta \leq \frac{16}{\boldsymbol{\rho}(0) \sinh (\boldsymbol{\rho}(0))} \int_{\mathcal{R}\left(r^{2}\right)} t \Delta \boldsymbol{\rho}^{2}\left(t e^{i \theta}\right) \frac{d t d \theta}{\left(1-t^{2}\right)^{2}}
$$

whenever $1 / 2 \leq r^{2}<1$. Using that for $0 \leq t<r^{2}$ the inequality

$$
1 \leq \frac{\log (r / t)}{\log (1 / r)}
$$


holds, we find

$$
\begin{aligned}
\int_{\mathcal{R}\left(r^{2}\right)} t \Delta \rho^{2}\left(t e^{i \theta}\right) \frac{d t d \theta}{\left(1-t^{2}\right)^{2}} & \leq\left(\log \frac{1}{r}\right)^{-1} \int_{\mathcal{R}\left(r^{2}\right)} t \log \frac{r}{t} \Delta \rho^{2}\left(t e^{i \theta}\right) \frac{d t d \theta}{\left(1-t^{2}\right)^{2}} \\
& \leq\left(\log \frac{1}{r}\right)^{-1} \int_{\mathcal{R}(r)} t \log \frac{r}{t} \Delta \rho^{2}\left(t e^{i \theta}\right) \frac{d t d \theta}{\left(1-t^{2}\right)^{2}} .
\end{aligned}
$$

Applying (13) to estimate from below the left hand side in the previous inequality, we conclude

$$
\int_{E_{r^{2}}} \Theta^{2}\left(r^{2} e^{i \theta}\right) d \theta \leq \frac{16\left(\log \frac{1}{r}\right)^{-1}}{\boldsymbol{\rho}(0) \sinh (\boldsymbol{\rho}(0))} \int_{\mathcal{R}(r)} t \log \frac{r}{t} \Delta \boldsymbol{\rho}^{2}\left(t e^{i \theta}\right) \frac{d t d \theta}{\left(1-t^{2}\right)^{2}}
$$

The following lemma is the main result of this section.

Lemma 2.1. For every $r \in\left[\frac{1}{\sqrt{2}}, 1\right)$ the estimate

$$
\int_{E_{r^{2}}} \Theta^{2}\left(r^{2} e^{i \theta}\right) d \theta \leq \frac{24 \pi K^{2}(\rho(r)+4)^{2}\left(\log \frac{1}{r}\right)^{-1}}{\sinh (\boldsymbol{\rho}(0))}
$$

holds.

Proof. Using the Green's formula (3), we estimate the integral on the right hand side in (14) and get

$$
\int_{E_{r^{2}}} \Theta^{2}\left(r^{2} e^{i \theta}\right) d \theta \leq \frac{8 \pi\left(\log \frac{1}{r}\right)^{-1}}{\boldsymbol{\rho}(0) \sinh (\boldsymbol{\rho}(0))}\left(\frac{1}{2 \pi} \int_{-\pi}^{\pi} \boldsymbol{\rho}^{2}\left(r e^{i \theta}\right) d \theta-\boldsymbol{\rho}^{2}(0)\right) .
$$

From Lemma 1.1 we conclude for every $(r, \theta) \in[0,1] \times(-\pi, \pi]$, the inequality

$$
\boldsymbol{\rho}^{2}\left(r e^{i \theta}\right) \leq \boldsymbol{\rho}^{2}(0)+3 K^{2} \boldsymbol{\rho}(0)(\rho(r)+4)^{2},
$$

holds. Replacing this into the previous inequality yields the lemma.

\section{Geometry of a $K$-quasiconformal Homeomorphism}

We show in this section that $\boldsymbol{\theta}\left(r e^{i \theta}\right)$ and $(\theta \circ g)\left(r e^{i \theta}\right)$ are very close to each other providing that $\theta \in U_{r}$ and that $\rho(r)$ is small comparing to $\boldsymbol{\rho}(0)$. This will give a lower bound for $\left|\boldsymbol{\theta}\left(r e^{i \theta}\right)\right|$ when $\theta \in U_{r}$.

3.1. The Set $U_{r}$. We define $U_{r}$ as follows.

Definition 3.1. For $0<r<1$, we let

$$
U_{r}=\left\{\theta \in(-\pi, \pi]: \boldsymbol{\rho}^{2}\left(r e^{i \theta}\right) \geq \boldsymbol{\rho}^{2}(0)-K^{2} \boldsymbol{\rho}(0)(\rho(r)+4)^{2}\right\} .
$$

Remark. Compare the definition of $U_{r}$ with the bound on the growth of $\rho^{2}(z)$ from Lemma 1.1 from Section 1. 
Lemma 3.1. For each $0 \leq r<1$, we have

$$
\left|U_{r}\right| \geq \frac{\pi}{2}
$$

where $\left|U_{r}\right|$ is the Lebesgue measure of $U_{r}$.

Proof. From the Green's formula (3) we find

$$
\boldsymbol{\rho}^{2}(0) \leq \frac{1}{2 \pi} \int_{-\pi}^{\pi} \boldsymbol{\rho}^{2}\left(r e^{i \theta}\right) d \theta .
$$

Using the definition of $U_{r}$ and the upper bound on $\rho^{2}$ from Lemma 1.1, we estimate the right hand side in (16) and derive

$$
\begin{aligned}
\boldsymbol{\rho}^{2}(0) & \leq\left(\boldsymbol{\rho}^{2}(0)-K^{2} \boldsymbol{\rho}(0)(\rho(r)+4)^{2}\right) \frac{\left(2 \pi-\left|U_{r}\right|\right)}{2 \pi} \\
& +\left(\boldsymbol{\rho}^{2}(0)+3 K^{2} \boldsymbol{\rho}(0)(\rho(r)+4)^{2}\right) \frac{\left|U_{r}\right|}{2 \pi} .
\end{aligned}
$$

This gives

$$
K^{2} \boldsymbol{\rho}(0)(\rho(r)+4)^{2} \leq \frac{\left|U_{r}\right|}{2 \pi} 4 K^{2} \boldsymbol{\rho}(0)(\rho(r)+4)^{2},
$$

which yields the lemma.

\subsection{Hölder Continuity of Quasiconformal Mappings and Applica-}

tions. Recall the following A-Priori bounds on the Hölder continuity constant of $g$. Namely, for every $K$-quasiconformal homeomorphism of $\mathbb{D}$ that fixes the origin 0 , the following holds:

$$
\frac{\left|z_{1}-z_{2}\right|^{K}}{16^{K}} \leq\left|g\left(z_{1}\right)-g\left(z_{2}\right)\right| \leq 16\left|z_{1}-z_{2}\right|^{\frac{1}{K}}, \quad z_{1}, z_{2} \in \mathbb{D} .
$$

This is a standard result and it can be found as Theorem 3.10 in [4].

Lemma 3.2. Let $\theta_{0} \in(-\pi, \pi]$ be such that $g\left(e^{i \theta_{0}}\right)=1 \in \mathbb{S}^{1}$. Then for $0<r<1$ the inequality

$$
\left|(\theta \circ g)\left(r e^{i \theta}\right)\right| \geq \frac{\left|e^{i \theta}-e^{i \theta_{0}}\right|^{K}}{16^{K}}-32 \arcsin \left((1-r)^{\frac{1}{K}}\right)
$$

holds.

Proof. By applying the triangle inequality twice, and since $(\theta \circ g)\left(e^{i \theta_{0}}\right)=0$, we have

$$
\begin{aligned}
& \left|(\theta \circ g)\left(r e^{i \theta}\right)\right|=\left|(\theta \circ g)\left(r e^{i \theta}\right)-(\theta \circ g)\left(r e^{i \theta_{0}}\right)\right| \\
& \geq\left|(\theta \circ g)\left(e^{i \theta}\right)-(\theta \circ g)\left(r e^{i \theta_{0}}\right)\right|-\left|(\theta \circ g)\left(r e^{i \theta}\right)-(\theta \circ g)\left(e^{i \theta}\right)\right| \\
& \geq\left|(\theta \circ g)\left(e^{i \theta}\right)-(\theta \circ g)\left(e^{i \theta_{0}}\right)\right|-\left|(\theta \circ g)\left(e^{i \theta_{0}}\right)-(\theta \circ g)\left(r e^{i \theta_{0}}\right)\right|-\left|(\theta \circ g)\left(r e^{i \theta}\right)-(\theta \circ g)\left(e^{i \theta}\right)\right| .
\end{aligned}
$$


Using the first inequality from (17), we estimate as follows

$$
\begin{aligned}
\left|(\theta \circ g)\left(e^{i \theta}\right)-(\theta \circ g)\left(e^{i \theta_{0}}\right)\right| & =\left|g\left(e^{i \theta}\right)-g\left(e^{i \theta_{0}}\right)\right| \\
& \geq \frac{\left|e^{i \theta}-e^{i \theta_{0}}\right|^{K}}{16^{K}} .
\end{aligned}
$$

By elementary Euclidean geometry, for $\alpha, \beta \in[-\pi, \pi]$, the inequality $\mid \alpha-$ $\beta \mid \leq 16 \arcsin (|\alpha-\beta| / 16)$ holds. Combining this with the second inequality from (17) we have

$\left|(\theta \circ g)\left(e^{i \theta_{0}}\right)-(\theta \circ g)\left(r e^{i \theta_{0}}\right)\right| \leq 16 \arcsin \frac{\left|g\left(e^{i \theta_{0}}\right)-g\left(r e^{i \theta_{0}}\right)\right|}{16} \leq 16 \arcsin \left((1-r)^{\frac{1}{K}}\right)$,

and likewise

$\left|(\theta \circ g)\left(r e^{i \theta}\right)-(\theta \circ g)\left(e^{i \theta}\right)\right| \leq 16 \arcsin \frac{\left|g\left(e^{i \theta}\right)-g\left(r e^{i \theta}\right)\right|}{16} \leq 16 \arcsin \left((1-r)^{\frac{1}{K}}\right)$.

Combining the previous 4 inequalities proves the lemma.

3.3. The next lemma provides an estimate from below of the value of the function $\left|\boldsymbol{\theta}\left(r e^{i \theta}\right)\right|$ when $\theta \in U_{r}$.

Lemma 3.3. Assume that $r \in[0,1)$ is such that

$$
\rho(r) \leq \frac{\sqrt{\boldsymbol{\rho}(0)}}{10 K}-4 .
$$

Then the estimate

$$
\left|\boldsymbol{\theta}\left(r e^{i \theta}\right)\right| \geq \frac{\left|e^{i \theta}-e^{i \theta_{0}}\right|^{K}}{16^{K}}-32 \arcsin \left((1-r)^{\frac{1}{K}}\right)-\arccos \left(1-e^{5 K} e^{-\frac{\rho(r)}{K}}\right)
$$

holds for every $\theta \in U_{r}$.

Proof. We observe the triangle inequality

$$
\left|\boldsymbol{\theta}\left(r e^{i \theta}\right)\right| \geq\left|(\theta \circ g)\left(r e^{i \theta}\right)\right|-\left|\boldsymbol{\theta}\left(r e^{i \theta}\right)-(\theta \circ g)\left(r e^{i \theta}\right)\right| .
$$

The lemma will follow from Lemma 3.2 and the inequality (23) below which we establish in the remainder of this proof.

Consider the geodesic triangle with vertices $0, g\left(r e^{i \theta}\right), f\left(r e^{i \theta}\right)$ and we let $\alpha$ denote the angle at the vertex 0 . Then

$$
\boldsymbol{\theta}\left(r e^{i \theta}\right)-(\theta \circ g)\left(r e^{i \theta}\right)=\alpha .
$$

We estimate $|\alpha|$ from above.

We let $z=r e^{i \theta}$. By the cosh formula from Hyperbolic Geometry we have

$$
\cosh ((\rho \circ g)(z)) \cosh (\boldsymbol{\rho}(z))-\sinh ((\rho \circ g)(z)) \sinh (\boldsymbol{\rho}(z)) \cos \alpha
$$

$$
=\cosh (d(g(z), f(z)))
$$


Either $|\alpha|>\pi / 2$ (in which case the lemma holds) or the left hand side in (19) is greater or equal to

$$
\begin{aligned}
\cosh ((\rho \circ g)(z)) & \cosh (\boldsymbol{\rho}(z))-\cosh ((\rho \circ g)(z)) \cosh (\boldsymbol{\rho}(z)) \cos \alpha \\
& =\cosh ((\rho \circ g)(z)) \cosh (\boldsymbol{\rho}(z))(1-\cos \alpha) \\
& \geq \exp ((\rho \circ g)(z)+\boldsymbol{\rho}(z))(1-\cos \alpha) .
\end{aligned}
$$

The right hand side in (19) is smaller than $2 \exp (\boldsymbol{\rho}(0))$ (remember, we assume $\boldsymbol{\rho}(0)>1)$. Replacing this back into (19) we get

$$
\exp ((\rho \circ g)(z)+\boldsymbol{\rho}(z))(1-\cos \alpha) \leq 2 \exp (\boldsymbol{\rho}(0))
$$

As we mentioned before, every $K$-quasiconformal homeomorphism is a $(K, 3 K)$ quasi-isometry in terms of the hyperbolic metric. Therefore, the inequality

$$
(\rho \circ g)(z) \geq \frac{\rho(r)}{K}-3 K
$$

holds. Assuming $\theta \in U_{r}$ yields

$$
\boldsymbol{\rho}(z) \geq \sqrt{\boldsymbol{\rho}^{2}(0)-K^{2} \boldsymbol{\rho}(0)(\rho(r)+4)^{2}} .
$$

From the upper bound in (18) we find that $K^{2}(\rho(r)+4)^{2}<\boldsymbol{\rho}(0)$ and obtain

$$
\boldsymbol{\rho}(z)>\boldsymbol{\rho}(0)-1 .
$$

Feeding (21) and (22) back into (20) yields

So

$$
1-\cos \alpha \leq 2 e^{3 K} \exp \left(1-\frac{\rho(r)}{K}\right) \leq e^{5 K} e^{-\frac{\rho(r)}{K}},
$$

$$
|\alpha| \leq \arccos \left(1-e^{5 K} e^{-\frac{\rho(r)}{K}}\right)
$$

which proves the lemma.

3.4. The following is a corollary of Lemma 3.3 and it will be used in the proof of Theorem 1.1 below.

Lemma 3.4. There exist constants $p, q>0$ and $0<r_{0}<1$, that depend only on $K$, such that for $r_{0}<r<1$, and providing that

$$
100 K^{2}(\rho(r)+4)^{2}=\Psi_{1}(r, K) \leq \boldsymbol{\rho}(0),
$$

the estimate

$$
\int_{T}\left(\left|\boldsymbol{\theta}\left(r e^{i \theta}\right)\right|-\psi\left(e^{i \theta}\right)\right)^{2} d \theta \geq q
$$

holds for every measurable set $T \subset U_{r}$ of measure at least $\frac{\pi}{4}$ and every measurable function $\psi: \mathbb{S}^{1} \rightarrow \mathbb{R}$ such that $\|\psi\|_{\infty}<p$. 
Proof. First, choose $r_{0}=r_{0}(K)$ such that

$$
\frac{1}{2} \frac{\left|e^{i \theta}-e^{i \theta_{0}}\right|^{K}}{16^{K}}>32 \arcsin \left(\left(1-r_{0}\right)^{\frac{1}{K}}\right)+\arccos \left(1-e^{5 K} e^{-\frac{\rho(r)}{K}}\right) .
$$

Then from Lemma 3.3 we have

$$
\left|\boldsymbol{\theta}\left(r e^{i \theta}\right)\right| \geq \frac{1}{2} \frac{\left|e^{i \theta}-e^{i \theta_{0}}\right|^{K}}{16^{K}}, \quad \theta \in U_{r},
$$

for $r>r_{0}$. This implies the inequality

$$
\left|\boldsymbol{\theta}\left(r e^{i \theta}\right)\right| \geq c>0, \quad \theta \in U_{r} \text { and }\left|\theta-\theta_{o}\right| \geq \frac{\pi}{16},
$$

for $r>r_{0}$, where $c=c(K)>0$ depends only on $K$.

Suppose that $\psi: \mathbb{S}^{1} \rightarrow \mathbb{R}$ is a measurable function. Suppose $T \subset U_{r}$ and let $\widehat{T}=T \cap\left\{e^{i \theta}:\left|\theta-\theta_{0}\right| \geq \frac{\pi}{16}\right\}$. Then

$$
\begin{aligned}
\int_{T}\left(\left|\boldsymbol{\theta}\left(r e^{i \theta}\right)\right|-\psi\left(e^{i \theta}\right)\right)^{2} d \theta & \geq \int_{\widehat{T}}\left(\left|\boldsymbol{\theta}\left(r e^{i \theta}\right)\right|-\psi\left(e^{i \theta}\right)\right)^{2} d \theta \\
& \geq|\widehat{T}|\left(c(K)-\|\psi\|_{\infty}\right)^{2} .
\end{aligned}
$$

Since by the assumption $|T| \geq \frac{\pi}{4}$, we conclude $|\widehat{T}| \geq \frac{\pi}{8}$ and the estimate

$$
\int_{T}\left(\left|\boldsymbol{\theta}\left(r e^{i \theta}\right)\right|-\psi\left(e^{i \theta}\right)\right)^{2} d \theta \geq \frac{\pi}{8}\left(c(K)-\|\psi\|_{\infty}\right)^{2}
$$

follows.

We set $p=\frac{c(K)}{2}$ and $q=\frac{\pi}{32} c(K)^{2}$, and verify that the inequality in this lemma holds for our choice of $p, q$ and $r_{0}$.

\section{Estimating The Distance Between $f$ and $g$ in Terms of$$
\|\operatorname{Hopf}[f]\|_{\infty}
$$

In this section we bound by above the partial derivative $|\partial f|$ in terms of the norm of Hopf $[f]$. It turns out that the inequality

$$
\sup _{z \in \mathbb{D}}|\partial f|(z) \leq C \sup _{z \in \mathbb{D}} d(f(z), g(z))+B(K)
$$

holds, where $C$ is a universal constant and $B(K)$ depends only on $K$. The proof below relies on some very standard facts about Hopf Differentials, but we point out that the Lemma 4.3 can be proved using the Chang's Lemma (I thank the referee for pointing this out). 
4.1. In [16] and [17] M. Wolf gave a comprehensive analysis of the degeneration of harmonic mappings whose Hopf's differentials blow up. Although initially he considered the case of closed Riemann surfaces his analysis is local in nature and has been widely deployed in studying harmonic maps between non-compact surfaces. The following lemma follows from Wolf's work.

Lemma 4.1. There exists a universal constant $c>0$ such that for every harmonic quasiconformal map $f: \mathbb{D} \rightarrow \mathbb{D}$ there exist points $z_{0}, z_{1} \in \mathbb{D}$, with the properties $d\left(z_{0}, z_{1}\right)<1$, and

$$
d\left(f\left(z_{0}\right), f\left(z_{1}\right)\right)>c \sqrt{\|\operatorname{Hopf}[f]\|_{\infty}} .
$$

Proof. Let Hopf $[f]=\Phi$ and write $\Phi=t \Phi_{0}$, where $t=\|\Phi\|_{\infty}$ and $\left\|\Phi_{0}\right\|_{\infty}=$ 1 . Let $z_{0} \in \mathbb{D}$ be a point where $\sigma^{-2}\left(z_{0}\right)\left|\Phi_{0}\left(z_{0}\right)\right|=1 / 2$. First we state and proof the following claim.

Claim 1. There exists a universal (independent of $\Phi_{0}$ ) constant $\delta>0$ such that $\Phi_{0}$ has no zeroes in the disk of hyperbolic radius $\delta$ centered at $z_{0}$.

Proof. For the purposes of this proof only we may assume that $z_{0}=0$. Then $\left|\Phi_{0}(z)\right| \leq \sigma^{2}(z)=4 /\left(1-|z|^{2}\right)^{2}$, and $\Phi_{0}(0)=\frac{1}{2}$, so for $\Phi \in \operatorname{BQD}(\mathbb{D})$ the functions $\Phi_{0}$ constitute a normal family on $\mathbb{D}$. Since $\Phi_{0}(0) \neq 0$, it follows that $\Phi_{0}(z) \neq 0$ in some neighborhood of 0 . Therefore, there exists a universal (independent of $\Phi_{0}$ ) constant $\delta>0$ such that $\Phi_{0}$ has no zeroes in the disk of hyperbolic radius $\delta$ centered at 0 .

Let $z_{1}$ be a point in this disc that lies on the horizontal trajectory of $\Phi_{0}$ that passes through 0 , and such that

$$
d\left(0, z_{1}\right)=\min \left\{\frac{\delta}{2}, 1\right\} .
$$

Let $\gamma$ denote the arc of this horizontal trajectory that connects 0 and $z_{1}$. It was shown in [16] (see (b) in Corollary 4.5 in [16]) that for some universal constant $c>0$ we have

$$
l_{\sigma}(f(\gamma))>\frac{c}{2} \sqrt{t}
$$

where $l_{\sigma}(f(\gamma))$ denotes the hyperbolic length of $f(\gamma)$. In [17] (see the last claim in Lemma 2.2 in [17]) it was shown that the geodesic curvature of $f(\gamma)$ uniformly tends to 0 when $t \rightarrow \infty$. This implies that for every $\epsilon>0$ there exists $t_{0}=t_{0}(\epsilon)$ (in particular, $t_{0}$ is independent of $\Phi_{0}$ ) such that for $t>t_{0}$ the ratio between the lengths of $f(\gamma)$ and the geodesic arc with the same endpoints belongs to the interval $[1-\epsilon, 1+\epsilon]$. This yields the estimate

$$
d\left(f\left(z_{0}\right), f\left(z_{1}\right)\right)>c \sqrt{t}
$$

for $t$ large enough. The lemma is proved.

We can now estimate from below the distance between $f$ and $g$ in terms of the norm of $\operatorname{Hopf}[f]$. 
Lemma 4.2. There exists a constant $b(K) \geq 0$ such that

$$
\sup _{z \in \mathbb{D}} d(f(z), g(z)) \geq \frac{c}{2} \sqrt{\|\operatorname{Hopf}[f]\|_{\infty}}-b(K),
$$

where $c>0$ is the universal constant from Lemma 4.1.

Proof. Using the notation from the proof of Lemma 4.1 we note that there exists a constant $b(K)>0$ such that $d\left(g\left(z_{0}\right), g\left(z_{1}\right)\right)>b(K)$ (this follows from the fact that the distance between $z_{0}$ and $z_{1}$ is bounded below by the universal constant $\delta / 2$ and the assumption that $g$ is $K$-quasiconformal). Then

$$
d\left(f\left(z_{0}\right), f\left(z_{1}\right)\right) \leq d\left(f\left(z_{0}\right), g\left(z_{0}\right)\right)+d\left(g\left(z_{0}\right), g\left(z_{1}\right)\right)+d\left(g\left(z_{1}\right), f\left(z_{1}\right)\right),
$$

which we rewrite as

$$
d\left(f\left(z_{0}\right), g\left(z_{0}\right)\right)+d\left(g\left(z_{1}\right), f\left(z_{1}\right)\right) \geq d\left(f\left(z_{0}\right), f\left(z_{1}\right)\right)-d\left(g\left(z_{0}\right), g\left(z_{1}\right)\right),
$$

and so by Lemma 4.1

$$
\max \left\{d\left(f\left(z_{0}\right), g\left(z_{0}\right)\right), d\left(g\left(z_{1}\right), f\left(z_{1}\right)\right\} \geq \frac{c}{2} \sqrt{\|\operatorname{Hopf}[f]\|_{\infty}}-b(K) .\right.
$$

The lemma is proved

Let

In [15] T. Wan proved the inequality

$$
\partial f=\frac{(\sigma \circ f)}{\sigma} f_{z} .
$$

$$
1 \leq|\partial f|^{2}(z) \leq \frac{1+\sqrt{1+4\|\operatorname{Hopf}[f]\|_{\infty}^{2}}}{2} .
$$

Together with Lemma 4.2 this implies the following result.

Lemma 4.3. There exists a constant $B(K) \geq 0$ and a universal constant $C>0$, such that

$$
\sup _{z \in \mathbb{D}}|\partial f|(z) \leq C \sup _{z \in \mathbb{D}} d(f(z), g(z))+B(K)
$$

Proof. For some universal constant $B_{1}>0$ we have

$$
\frac{1+\sqrt{1+4\|\operatorname{Hopf}[f]\|_{\infty}^{2}}}{2} \leq\|\operatorname{Hopf}[f]\|_{\infty}+B_{1} .
$$

By Lemma 4.2 we have

$$
\|\operatorname{Hopf}[f]\|_{\infty} \leq\left(C \sup _{z \in \mathbb{D}} d(f(z), g(z))+B_{2}(K)\right)^{2}
$$

for a universal constant $C>0$ and a constant $B_{2}(K)>0$. Then for $B(K)=$ $B_{2}(K)+B_{1}+1$ we have

$$
\|\operatorname{Hopf}[f]\|_{\infty}+B_{1} \leq\left(C \sup _{z \in \mathbb{D}} d(f(z), g(z))+B(K)\right)^{2},
$$


which together with (24) and (25) proves the lemma.

\section{Proof of Theorem 1.1}

In the first subsection below we use Lemma 4.3 to find an upper bound for $\boldsymbol{\theta}\left(e^{i \theta} / 2\right)$ when $\theta \in E_{1 / 2}$. In the second subsection (again using Lemma 4.3 ) we show that the set $E_{r}$ has almost the full measure in $(-\pi, \pi]$ when $\boldsymbol{\rho}(0)$ is large enough. We then complete the proof of Theorem 1.1.

5.1. Estimating $\boldsymbol{\theta}$ on the Set $\left(0, \frac{1}{2}\right) \times E_{1 / 2}$. Recall that for $0<r<1$ the set $E_{r}$ is the set of $\theta \in(-\pi, \pi]$ such that

$$
\boldsymbol{\rho}\left(t e^{i \theta}\right) \geq \frac{\boldsymbol{\rho}(0)}{2}, \quad 0 \leq t \leq r .
$$

Lemma 5.1. Let $\epsilon>0$. There exists $L=L(K, \epsilon)>0$ such that for $\boldsymbol{\rho}(0)>L$ and for every $\theta \in E_{1 / 2}$ we have

$$
\left|\boldsymbol{\theta}\left(e^{i \theta} / 2\right)\right| \leq \epsilon
$$

Proof. Let $\gamma=\left[0, \frac{1}{2}\right]$ be the geodesic arc in $\mathbb{D}$. Then by Lemma 4.3 we have

$$
l_{\sigma}(f(\gamma)) \leq \int_{0}^{\frac{1}{2}} 2|\partial f|\left(t e^{i \theta}\right) \frac{2 d t}{1-t^{2}} \leq 4 \log 3(C(\boldsymbol{\rho}(0)+1)+B(K)),
$$

where $C>0$ and $B(K)>0$ are constants from Lemma 4.3 and $l_{\sigma}(f(\gamma))$ denotes the hyperbolic length.

On the other hand, since $\theta \in E_{1 / 2}$, the curve $f(\gamma)$ stays outside the geodesic disc of radius $\boldsymbol{\rho}(0) / 2$ centered at 0 . Therefore, the estimate

$$
\left|\boldsymbol{\theta}\left(e^{i \theta} / 2\right)-\boldsymbol{\theta}(0)\right| \sinh (\boldsymbol{\rho}(0) / 2) \leq l_{\sigma}(f(\gamma))
$$

holds (recall that the volume element of the hyperbolic metric in the geodesic polar coordinates is $\left.d V=d \rho^{2}+\sinh (\rho) d \theta^{2}\right)$. Since $\boldsymbol{\theta}(0)=0$ (remember, we assume $f(0) \in(0,1))$, using (26) we get

$$
\left|\boldsymbol{\theta}\left(e^{i \theta} / 2\right)\right| \leq \frac{4 \log 3(C(\boldsymbol{\rho}(0)+1)+B(K))}{\sinh (\boldsymbol{\rho}(0) / 2)},
$$

and we choose $L=L(K, \epsilon)$ large enough so that the right hand side is smaller than $\epsilon$ for $\boldsymbol{\rho}(0)>L$.

5.2. The Measure of $E_{r}$. Consider the set $\mathcal{R}(r)=[0, r] \times(-\pi, \pi]$ with its Lebesgue measure. Let

$$
X_{r}=\left\{(t, \theta) \in \mathcal{R}(r): \boldsymbol{\rho}\left(t e^{i \theta}\right) \leq \frac{3 \boldsymbol{\rho}(0)}{4}\right\} .
$$


Proposition 5.1. There exists a constant $c=c(K)$ such that

$$
\left|X_{r}\right| \geq c(K)\left(1-r^{2}\right)\left(2 \pi-\left|E_{r}\right|\right),
$$

holds, where $\left|X_{r}\right|$ is the (two dimensional) Lebesgue measure of $X_{r} \subset \mathcal{R}(r)$ and $\left|E_{r}\right|$ the (one dimensional) Lebesgue measure of $E_{r} \subset(-\pi, \pi]$.

Proof. Suppose $\theta \notin E_{r}$. Then for some $0<t \leq r$ we have $\boldsymbol{\rho}\left(t e^{i \theta}\right)<\boldsymbol{\rho}(0) / 2$, so by continuity there exists the smallest number $0<t_{1} \leq r$ such that $\boldsymbol{\rho}\left(t_{1} e^{i \theta}\right)=\boldsymbol{\rho}(0) / 2$. Let $t_{2}>0$ be the largest number such that $t_{2}<t_{1}$ and $\boldsymbol{\rho}\left(t_{2} e^{i \theta}\right)=3 \boldsymbol{\rho}(0) / 4$ (such $t_{2}$ exists by continuity because $\boldsymbol{\rho}(0)$ is strictly greater than $\left.\boldsymbol{\rho}\left(t_{1} e^{i \theta}\right)\right)$. Observe the inequality $\boldsymbol{\rho}(0) / 4 \leq d\left(f\left(t_{1} e^{i \theta}\right), f\left(t_{2} e^{i \theta}\right)\right)$. This holds since $f\left(t_{1} e^{i \theta}\right)$ lives on the circle of hyperbolic radius $\boldsymbol{\rho}(0) / 2$ and $f\left(t_{2} e^{i \theta}\right)$ is on the circle of hyperbolic radius $3 \boldsymbol{\rho}(0) / 4$.

By construction, for each $t \in\left[t_{2}, t_{1}\right]$ we have $\boldsymbol{\rho}\left(t e^{i \theta}\right) \leq 3 \boldsymbol{\rho}(0) / 4$, that is $t e^{i \theta} \in X_{r}$. On the other hand, we have

$$
\begin{aligned}
\frac{\boldsymbol{\rho}(0)}{4} \leq d\left(f\left(t_{1} e^{i \theta}\right), f\left(t_{2} e^{i \theta}\right)\right) & \leq \int_{t_{1}}^{t_{2}} 2|\partial f|\left(t e^{i \theta}\right) \frac{2 d t}{1-t^{2}} \\
& \leq \frac{4\left|t_{2}-t_{1}\right|}{1-r^{2}}(C(\boldsymbol{\rho}(0)+1)+B(K)),
\end{aligned}
$$

where $C>0$ and $B(K)>0$ are constants from Lemma 4.3. This implies the estimate

$$
\left|t_{1}-t_{2}\right| \geq c(K)\left(1-r^{2}\right)
$$

for some constant $c(K)$ that depends only on $K$. Since for each $\theta \notin E_{r}$ the segment $\left[t_{2} e^{i \theta}, t_{1} e^{i \theta}\right]$ is contained in $X_{r}$, by the last inequality we obtain the bound

$$
\left|X_{r}\right|>c(K)\left(1-r^{2}\right)\left(2 \pi-\left|E_{r}\right|\right)
$$

which proves the lemma.

We can now show that $E_{r}$ has almost full measure when $\boldsymbol{\rho}(0)$ is large.

Lemma 5.2. There exist a constant $c=c(K)>0$ such that for every $0 \leq r<1$ that satisfies the condition

$$
\frac{24 r K^{2}(\rho(r)+4)^{2}}{c(K)\left(1-r^{2}\right)}=\Psi_{2}(r, K) \leq \boldsymbol{\rho}(0),
$$

the estimate

$$
\frac{7 \pi}{4} \leq\left|E_{r}\right|
$$

holds. 
Proof. The function $\boldsymbol{\rho}^{2}$ is subharmonic and (3) implies the inequality

$$
\rho^{2}(0) \leq \frac{1}{2 \pi} \int_{-\pi}^{\pi} \rho^{2}\left(t e^{i \theta}\right) d \theta
$$

for every $t \in[0,1)$. Integrating this inequality over the interval $[0, r]$ yields

$$
0 \leq \frac{1}{2 \pi} \int_{\mathcal{R}(r)}\left(\frac{\boldsymbol{\rho}^{2}\left(t e^{i \theta}\right)}{\boldsymbol{\rho}^{2}(0)}-1\right) d t d \theta .
$$

We split the integral on the right hand side into the sum of integrals over the sets $X_{r}$ and $\mathcal{R}(r) \backslash X_{r}$. This yields

$$
0 \leq \frac{1}{2 \pi} \int_{X_{r}}\left(\frac{\boldsymbol{\rho}^{2}\left(t e^{i \theta}\right)}{\boldsymbol{\rho}^{2}(0)}-1\right) d t d \theta+\frac{1}{2 \pi} \int_{\mathcal{R}(r) \backslash X_{r}}\left(\frac{\boldsymbol{\rho}^{2}\left(t e^{i \theta}\right)}{\boldsymbol{\rho}^{2}(0)}-1\right) d t d \theta .
$$

From Lemma 1.1 we conclude that the following bound holds for every $t e^{i \theta}$

$$
\frac{\boldsymbol{\rho}^{2}\left(t e^{i \theta}\right)}{\boldsymbol{\rho}^{2}(0)}-1 \leq 3 K^{2} \frac{(\rho(t)+4)^{2}}{\boldsymbol{\rho}(0)} .
$$

On the other hand, for $(r, \theta) \in X_{r}$ we have

$$
\frac{\rho^{2}\left(t e^{i \theta}\right)}{\rho^{2}(0)}-1 \leq-\frac{7}{16} \leq-\frac{1}{4}
$$

Using the last two inequalities we estimate the right hand side in (27) and get

which implies

$$
0 \leq-\frac{1}{4}\left|X_{r}\right|+\frac{3 K^{2}(\rho(r)+4)^{2}}{\rho(0)}\left(|\mathcal{R}(r)|-\left|X_{r}\right|\right),
$$

$$
\frac{1}{4}\left|X_{r}\right| \leq \frac{6 \pi r K^{2}(\rho(r)+4)^{2}}{\boldsymbol{\rho}(0)} .
$$

Here we used $\left(|\mathcal{R}(r)|-\left|X_{r}\right|\right) \leq|\mathcal{R}(r)|=2 \pi r$.

On the other hand, from Proposition 5.1 we have

$$
\left|X_{r}\right| \geq c(K)\left(1-r^{2}\right)\left(2 \pi-\left|E_{r}\right|\right),
$$

and replacing this in $(28)$ yields

$$
2 \pi-\frac{24 \pi r K^{2}(\rho(r)+4)^{2}}{c(K)\left(1-r^{2}\right) \boldsymbol{\rho}(0)} \leq\left|E_{r}\right| .
$$

Letting

$$
\boldsymbol{\rho}(0) \geq \frac{24 r K^{2}(\rho(r)+4)^{2}}{c(K)\left(1-r^{2}\right)}
$$

completes the proof. 
5.3. The Endgame. From Lemma 2.1 we have

$$
\int_{E_{r^{2}}} \Theta^{2}\left(r^{2} e^{i \theta}\right) d \theta \leq \frac{24 K^{2}(\rho(r)+4)^{2} \pi\left(\log \frac{1}{r}\right)^{-1}}{\sinh (\boldsymbol{\rho}(0))}
$$

for every $\theta \in E_{r}$ when $0<1 / \sqrt{2} \leq r<1$. It remains to find a positive lower bound for

$$
\int_{E_{r^{2}}} \Theta^{2}\left(r^{2} e^{i \theta}\right) d \theta=\int_{E_{r^{2}}}\left(\left|\boldsymbol{\theta}\left(r^{2} e^{i \theta}\right)\right|-\left|\boldsymbol{\theta}\left(e^{i \theta} / 2\right)\right|\right)^{2} d \theta .
$$

We make the following choices.

- Let

$$
r_{1}=r_{1}(K)=\max \left\{r_{0}(K), \frac{1}{\sqrt{2}}\right\}
$$

denote the constant from Lemma 3.4.

- Let $p=p(K)>0$ and $q=q(K)>0$ be the constants from Lemma 3.4, and let $L(K, p)$ be the corresponding constant from Lemma 5.1.

- In the remainder of the proof we assume that $\boldsymbol{\rho}(0)$ is big enough so that the inequality

$$
\boldsymbol{\rho}(0)>\max \left(L(K, p), \Psi_{1}\left(r_{1}, K\right), \Psi_{2}\left(r_{1}, K\right)\right)
$$

holds (otherwise we have obtained an upper bound on $\boldsymbol{\rho}(0)$ that depends only on $K$ and Theorem 1.1 would be proved). Here $\Psi_{1}(r, K)$ is the function from Lemma 3.4 and $\Psi_{2}(r, K)$ is the function from Lemma 5.2.

Given these choices, by Lemma 5.1 we have

$$
\left|\boldsymbol{\theta}\left(e^{i \theta} / 2\right)\right| \leq p, \quad \theta \in E_{\frac{1}{2}} .
$$

On the other hand, from Lemma 5.2 and Lemma 3.1 we conclude that $\left|U_{r_{1}^{2}} \cap E_{r_{1}^{2}}\right| \geq \pi / 4$. Thus, from Lemma 3.4 we derive

$$
q(K) \leq \int_{U_{r_{1}^{2}} \cap E_{r_{1}^{2}}}\left(\left|\boldsymbol{\theta}\left(r_{1}^{2} e^{i \theta}\right)\right|-\left|\boldsymbol{\theta}\left(e^{i \theta} / 2\right)\right|\right)^{2} d \theta,
$$

which yields

$$
q(K) \leq \int_{E_{r_{1}^{2}}}\left(\left|\boldsymbol{\theta}\left(r_{1}^{2} e^{i \theta}\right)\right|-\left|\boldsymbol{\theta}\left(e^{i \theta} / 2\right)\right|\right)^{2} d \theta,
$$

Replacing this into (29) yields

$$
\sinh (\boldsymbol{\rho}(0)) \leq \frac{24 K^{2}\left(\rho\left(r_{1}\right)+4\right)^{2} \pi\left(\log \frac{1}{r_{1}}\right)^{-1}}{q(K)} .
$$

Recall that $r_{1}=r_{1}(K)$. Theorem 1.1 is proved. 


\title{
REFERENCES
}

[1] F. Bonsante, J-M. Schlenker, Maximal surfaces and the universal Teichmüller space. Inventiones Mathematicae 182 (2010), no. 2, 279-333.

[2] S. Cheng, Liouville theorem for harmonic maps. Geometry of the Laplace operator pp. 147-151, Proc. Sympos. Pure Math., XXXVI, Amer. Math. Soc., Providence, R.I., 1980

[3] D. Epstein, A. Marden, V. Markovic, Quasiconformal homeomorphisms and the convex hull boundary. Ann. of Math. (2) 159 (2004), no. 1, 305-336.

[4] A. Fletcher, V. Markovic, Quasiconformal maps and Teichmller theory. Oxford Graduate Texts in Mathematics, 11. Oxford University Press, Oxford, 2007

[5] Z. Han, Remarks on the geometric behavior of harmonic maps between surfaces. Elliptic and parabolic methods in geometry (Minneapolis, MN, 1994), 5766, A K Peters, Wellesley, MA, 1996.

[6] R. Hardt, M. Wolf, Harmonic extensions of quasiconformal maps to hyperbolic space. Indiana Univ. Math. J. 46 (1997), no. 1, 155-163.

[7] P. Li, L. Tam, Uniqueness and regularity of proper harmonic maps. Annals of Math. (2) 137 (1993), no. 1, 167-201.

[8] P. Li, L. Tam, Uniqueness and regularity of proper harmonic maps II. Indiana Univ. Math. J. 42 (1993), no. 2, 591-635.

[9] V. Markovic, Harmonic diffeomorphisms of noncompact surfaces and Teichmüller spaces. J. London Math. Soc. (2) 65 (2002), no. 1, 103114.

[10] V. Markovic, Harmonic maps between 3-dimensional hyperbolic spaces. Inventiones mathematicae, 199 (2015), 3, 921-951

[11] R. Schoen, The role of harmonic mappings in rigidity and deformation problems. Complex geometry (Osaka, 1990), 179-200, Lecture Notes in Pure and Appl. Math., 143, Dekker, New York, 1993

[12] R. Schoen, S.T. Yau, Lectures on harmonic maps. Conference Proceedings and Lecture Notes in Geometry and Topology, II. International Press, Cambridge, MA, 1997.

[13] M. Stoll, Harmonic Function Theory of Real Hyperbolic Space. preprint, see www.math.sc.edu/people/faculty/stoll/hyperbolic.pdf

[14] L. Tam, T. Wan, Quasi-conformal harmonic diffeomorphism and the universal Teichmüller space. J. Differential Geom. 42 (1995), no. 2, 368410.

[15] T. Wan, Constant mean curvature surface, harmonic maps, and universal Teichmüller space. J. Differential Geom. 35 (1992), no. 3,

[16] M. Wolf, The Teichmller theory of harmonic maps. Journal Differential Geom. 29 (1989), no. 2, 449479.

[17] M. Wolf, High energy degeneration of harmonic maps between surfaces and rays in Teichmller space. Topology 30 (1991), no. 4, 517540

\author{
Department of Mathematics \\ California Institute of Technology \\ PASADENA, CA 91125, USA \\ E-mail address: markovic@caltech.edu
}

